\section{GERAÇÃO DE MODELO DIGITAL DE TERRENO POR MEIO DA FILTRAGEM DE DADOS OBTIDOS POR VARREDURA A LASER UTILIZANDO UM MÉTODO BASEADO NO PRINCÍPIO DA DECLIVIDADE}

\section{DIGITAL TERRAIN MODEL GENERATION FROM SLOPE BASED FILTERING METHOD OF LASER SCANNING DATA}

Wallace Felipe Francisco Cardoso ${ }^{1}$, Francisco Assis da Silva ${ }^{2}$, Mário Augusto Pazoti ${ }^{2}$

${ }^{1}$ Discente da Faculdade de Informática da UNOESTE. ${ }^{2}$ Docente da Faculdade de Informática da UNOESTE.

RESUMO - Devido aos avanços na tecnologia dos sistemas de varredura a laser, pesquisas em vários países foram iniciadas com o objetivo de explorar os recentes recursos tecnológicos desenvolvidos. O resultado obtido pelos sistemas de varredura a laser possibilitou o detalhamento das características de regiões e a atualização dos modelos de territórios nacionais de muitos países. Os dados gerados pela varredura a laser correspondem ao conjunto de pontos de altimetria da superfície terrestre. Esse conjunto de pontos é denominado como Modelo Digital de Elevação. A derivação desse modelo contendo apenas características de terreno é denominada Modelo Digital de Terreno. Entretanto, os Modelos Digitais de Terreno não podem ser facilmente gerados por varredura a laser. Em geral, os Modelos Digitais de Terreno são derivados dos Modelos Digitais de Elevação com uso de métodos de filtragem. O objetivo deste trabalho é adaptar um método de filtragem de Modelos Digitais de Elevação e apresentar uma análise dos resultados obtidos.

Palavras-chave: modelo digital de terreno; varredura a laser; filtragem de dados laser; grade regular.

ABSTRACT - Due the advances in the technology of laser scanning systems, research in several countries have been initiated with the aim of exploring the recently technological resources developed. The result obtained by laser scanning systems provided the details of regions features and update many of the national territories models. The data generated by laser scanning match the altimetry data set named as Digital Elevation Model. This model derivation, when containing only terrain features, is called Digital Terrain Model. However, the Digital Terrain Models cannot be easily generated by laser scanning. In general, the Digital Terrain Models are derived from Digital Elevation Models applying filtering methods. This work objective is to adapt a Digital Elevation Model filtering method and discuss the results obtained.

Keywords: digital terrain model; scanning laser, filtering laser data; regular grid. 


\section{INTRODUÇÃO}

Os sistemas de varredura a laser têm se mostrando cada vez mais precisos quanto à obtenção dos dados de altimetria da superfície terrestre. Por esse motivo, vários países utilizaram-se destes dados para produzir ou atualizar os detalhes das regiões de seu território nacional. Embora existam alguns tipos de sistemas de varredura a laser conhecidos, os dados gerados por sistemas aerotransportados foram bastante explorados em pesquisas recentes. Tal sistema possui a característica da aquisição de dados em larga escala, de uma área de grande extensão territorial, em um curto espaço de tempo (VOSSELMAN, 2000).

Toda a mensuração dos pontos coletados pela varredura é feita de acordo com uma série de parâmetros do sistema. Os componentes principais do sistema de varredura a laser são: a unidade de emissão de feixes laser e de captura da reflexão dos feixes laser, a unidade inercial espacial, e o sistema de posicionamento global (FAZAN; DAL POZ, 2011).

O funcionamento básico da varredura a laser consiste na emissão de feixes de laser contra a superfície terrestre. Todo feixe de laser disparado tem um tempo entre a emissão e a captura de sua reflexão. Com base no tempo e na orientação do raio é determinada a distância dos pontos entre o sistema de varredura e a superfície terrestre. Um feixe de laser emitido contra a superfície terrestre pode gerar mais de uma única reflexão. Isso geralmente ocorre quando o feixe disparado consegue ultrapassar a superfície do primeiro obstáculo encontrado, como ilustrado na Figura 1. A segunda reflexão é muito útil, por exemplo, em áreas de vegetação, em que se é possível obter pontos que representam solo terrestre. Porém, em áreas urbanas, a rigidez dos materiais que as compõem impossibilita o retorno de mais de uma reflexão de luz para um único feixe (CENTENO; MITISHITA, 2007).

É característica de áreas urbanas a ocorrência de muitas perdas de informação durante a varredura. Devido à orientação dos feixes, ocorrem omissões de superfícies de objetos durante a coleta dos dados de altimetria (FAZAN; DAL POZ, 2011). 


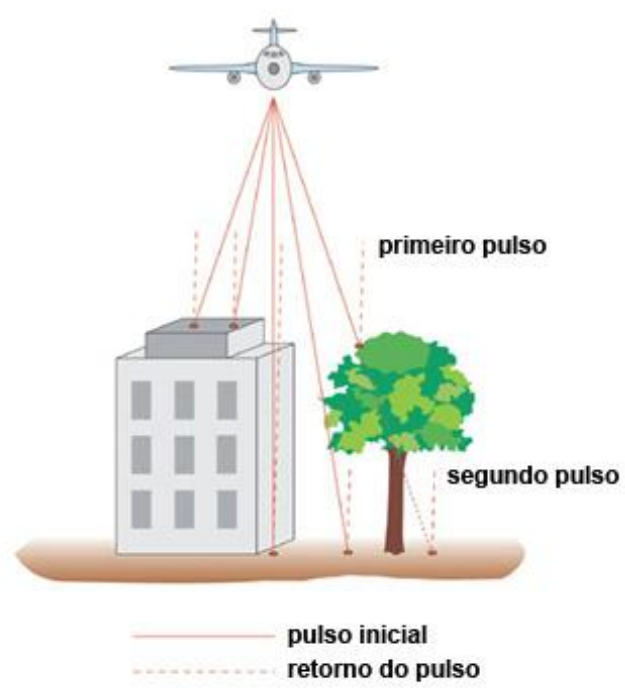

Figura 1. Exemplo do processo de varredura a laser por sistema aerotransportado. Adaptado de Terra Imaging (2013).

O conjunto de pontos gerados pela varredura a laser de uma superfície é chamado de Modelo Digital de Elevação (MDE). Durante a varredura podem ser coletados pontos de diferentes objetos, como: edificações, árvores, carros, etc. (CENTENO; MITISHITA, 2007). É importante conceituar as diferenças entre os Modelos Digitais de Elevação e os Modelos Digitais de Terreno (MDT). Ambos os modelos são formados por um conjunto de pontos, porém, em MDEs os pontos representam a elevação da superfície (prédios, árvores, carros, etc.). Por outro lado, em MDTs os pontos representam apenas características inerentes ao terreno (solo), como terra e água (ELMIRO et al., 2012; FAZAN; DAL POZ, 2011).
Os MDTs são muito utilizados na geração de ortofotos e mapas topográficos, como também no estudo das características do solo de regiões. Barbosa e Silva (1999) destacam o uso dos MDTs em algumas áreas de Engenharia, como: Engenharia Civil, Engenharia Cartográfica e Engenhara Militar.

O objetivo neste trabalho é estudar os métodos de filtragem conhecidos e desenvolver de um método de filtragem de MDE, aproveitando-se dos conceitos já existentes sobre filtragem de Modelos Digitais de Elevação.

A Seção 2 discute os conceitos básicos para o entendimento deste documento. A Seção 3 apresenta alguns trabalhos relacionados à pesquisa. A Seção 4 apresenta o objetivo da pesquisa e a motivação para a sua realização, assim como alguns detalhes 
importantes. A Seção 5 apresenta como foi feito o experimento utilizado para testar o método elaborado. A Seção 6 discute e apresenta a análise, a partir dos resultados obtidos. A Seção 7 apresenta as conclusões sobre o método proposto e considerações sobre o futuro dessa pesquisa.

\section{CONCEITOS BÁSICOS}

A Seção apresenta alguns conhecimentos básicos para o entendimento da pesquisa. A seção 2.1 apresenta os conceitos do princípio da filtragem proposto por Vosselman (2000). A Seção 2.2 apresenta o procedimento de geração de grades regulares com uso de interpolação por média simples, com base na nuvem de pontos gerada pela varredura laser. A Seção 2.3 apresenta conceitos de suavização de ruídos em processamento de sinais, posteriormente aplicados na grade regular. A Seção 2.4 apresenta o algoritmo proposto para filtragem de MDEs.

\subsection{O Princípio da Filtragem de Vosselman}

O princípio da filtragem proposto por Vosselman (2000) foi proposto como uma definição básica para a eliminação de pontos que não pertencentes ao terreno, em função da diferença de altura entre dois pontos adjacentes.
Seja A o conjunto de pontos do MDE, e $\Delta h_{\max }(d)$ a função que define a altura máxima permitida entre dois pontos adjacentes, a geração do MDT é dada pela Equação 1. Assim, todos os pontos cujas diferenças de altura entre estes e seus adjacentes forem maior que $\Delta h_{\max }(d)$, são desconsiderados do MDT.

$$
\begin{aligned}
M D T=\left\{p_{i} \in\right. & A \mid \forall p_{j} \in A \\
& : h_{p_{i}}-h_{p_{j}} \\
\leq & \left.\Delta h_{\max }\left(d\left(p_{i}, p_{j}\right)\right)\right\}
\end{aligned}
$$

Se $d$ for uma constante, devem-se apenas considerar os pontos com diferenças de distância de no máximo $d$ unidades. Vosselman (2000) apresenta uma derivação da função de filtragem com base no conhecimento sobre as características de declívio do terreno. A função sintética supõe que a declividade da área da superfície terrestre não seja tão íngreme, assim dizendo, $30 \%$.

Logo, em função da distância, a função sintética considera que a diferença máxima de altura deve ser igual a $30 \%$ da distância entre os pontos, como mostra a Equação 2.

$$
\Delta h_{\max }(d)=0.3 d
$$


2.2 Classificação dos Pontos em Grade Regular com Interpolador de Média Simples

O processo de classificação dos pontos tridimensionais é essencial para a aplicação de alguns métodos e técnicas de filtragem. Há um acervo com muitas técnicas de filtragem, oriundas do processamento de sinais. No entanto, estas requerem que os dados estejam organizados em grade (VOSSELMAN, 2000).

Dentre as formas de se gerar uma grade regular, encontra-se a interpolação por média móvel. A fórmula geral, dada por Felgueiras (2012), é mostrada na Equação 3.

$$
z_{i}=\frac{\sum_{j=1}^{n} w_{i j} z_{i j}}{\sum_{j=1}^{n} w_{i j}}
$$

Nesse caso, z corresponde à

coordenada $p_{z}$ dos pontos tridimensionais $p_{x y z}, w$ é um fator de ponderação e $z$ é o valor de cota, sendo $z_{i}$ os valores de cota da grade, e $z_{i j}$ os valores de cota da amostra vizinha ao ponto $i$.

A ideia da interpolação é estimar os valores de cota da grade com base em outra amostra, como ilustrado na Figura 2, em que o conjunto irregularmente espaçado (Figura 2a) é acomodado em um conjunto regularmente espaçado (Figura $2 b$ ).

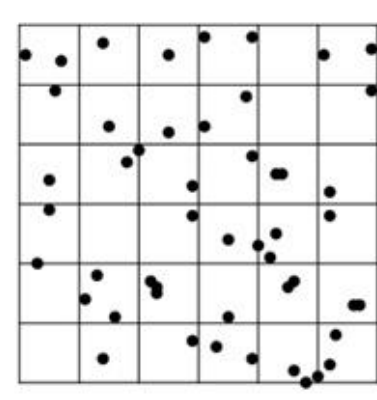

(a) irregular

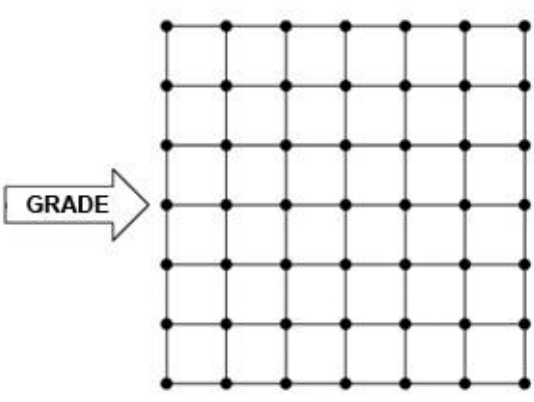

(b) regular

Figura 2. Exemplo de engradamento de uma amostra irregular de pontos (a) para uma amostra regular de pontos (b). Adaptado de GDAL (2012).

A interpolação de média simples é derivada da fórmula geral, em que o valor de cota $z$ da grade é igual à média aritmética da amostra vizinha (FELGUEIRAS, 2012). Portanto, o fator de ponderação nesse caso é igual a 1. As Equações 4 e 5 apresentam a fórmula geral com o fator de ponderação igual a 1. 


$$
\begin{aligned}
z_{i} & =\frac{\sum_{j=1}^{n} 1 z_{i j}}{\sum_{j=1}^{n} 1} \\
z_{i} & =\frac{\sum_{j=1}^{n} z_{i j}}{n}
\end{aligned}
$$

\subsection{Suavização de Ruídos com Filtro da}

\section{Mediana}

Alguns métodos de filtragem, como a função sintética de Vosselman (2000), por exemplo, consideram a amostra de pontos ausente de ruídos. Todavia, é possível que, após a geração da grade regular, sejam apresentados alguns pontos com valores de cota $z$ com uma diferenciação muito alta dos valores de cota $z$ da amostra vizinha.

Uma solução para isso foi obtida com a aplicação do filtro da mediana, que é um filtro estatístico não linear usado para reduzir ruídos, com uma considerável redução de borramento (GONZALEZ; WOODS, 1993). Por ser um método tradicional em Processamento de Imagens, realizou-se a adaptação para a grade regular considerando que cada elemento da grade corresponde a um ponto de coordenadas tridimensionais $(x$, $y, z)$, sendo z o valor de cota da grade.

A ideia básica do filtro de mediana é obter o elemento central de um subconjunto de pontos na vizinhança de um ponto $p$ e defini-lo como elemento de grade, substituindo o ponto $p$ pelo elemento central. Na Figura 3 é mostrado um exemplo da aplicação do filtro de mediana.

A aplicação do filtro da mediana na grade regular de pontos planialtimétricos tem por objetivo a eliminação de possíveis imperfeições na altimetria do MDS, sendo considerados como elementos os valores de cota $z$ da grade.

\begin{tabular}{|c|c|c|c|}
\hline 5 & 5 & 5 & 5 \\
\hline 5 & 5 & 59 & 5 \\
\hline 5 & 5 & 5 & 5 \\
\hline 5 & 5 & 5 & 5 \\
\hline
\end{tabular}

(a)

\begin{tabular}{|c|c|c|c|}
\hline 5 & 5 & 5 & 5 \\
\hline 5 & 5 & 59 & 5 \\
\hline 5 & 5 & 5 & 5 \\
\hline 5 & 5 & 5 & 5 \\
\hline
\end{tabular}

$(5,5,5,5,5,5,5,5,59)$

(b)

\begin{tabular}{|l|l|l|l|}
\hline 5 & 5 & 5 & 5 \\
\hline 5 & 5 & 5 & 5 \\
\hline 5 & 5 & 5 & 5 \\
\hline 5 & 5 & 5 & 5 \\
\hline
\end{tabular}

(c)

Figura 3. Exemplo da aplicação do filtro da mediana. Nos passos: (a) matriz com o valor 59 como ruído e ponto de origem; (b) aplicação do filtro em vizinhança $3 \times 3$, onde o elemento central da amostra ordenada é o valor 5; (c) resultado final, substituindo o ponto origem 59 pelo ponto 5. 


\subsection{O Algoritmo de Filtragem de MDE}

Segundo Vosselman (2000), a altura máxima permitida pela função $\Delta h_{\max }(d)$ cresce proporcionalmente à distância $d$. $\mathrm{O}$ processo de filtragem proposto nesse artigo é baseado nessa afirmação.
A ideia do filtro, depois de identificado um ponto não pertencente ao terreno, é eliminar a sequência de pontos não pertencentes ao terreno, subsequente ao ponto identificado, em uma direção definida, com base no na inclinação do terreno, como ilustrado nas Figuras 4 e 5.

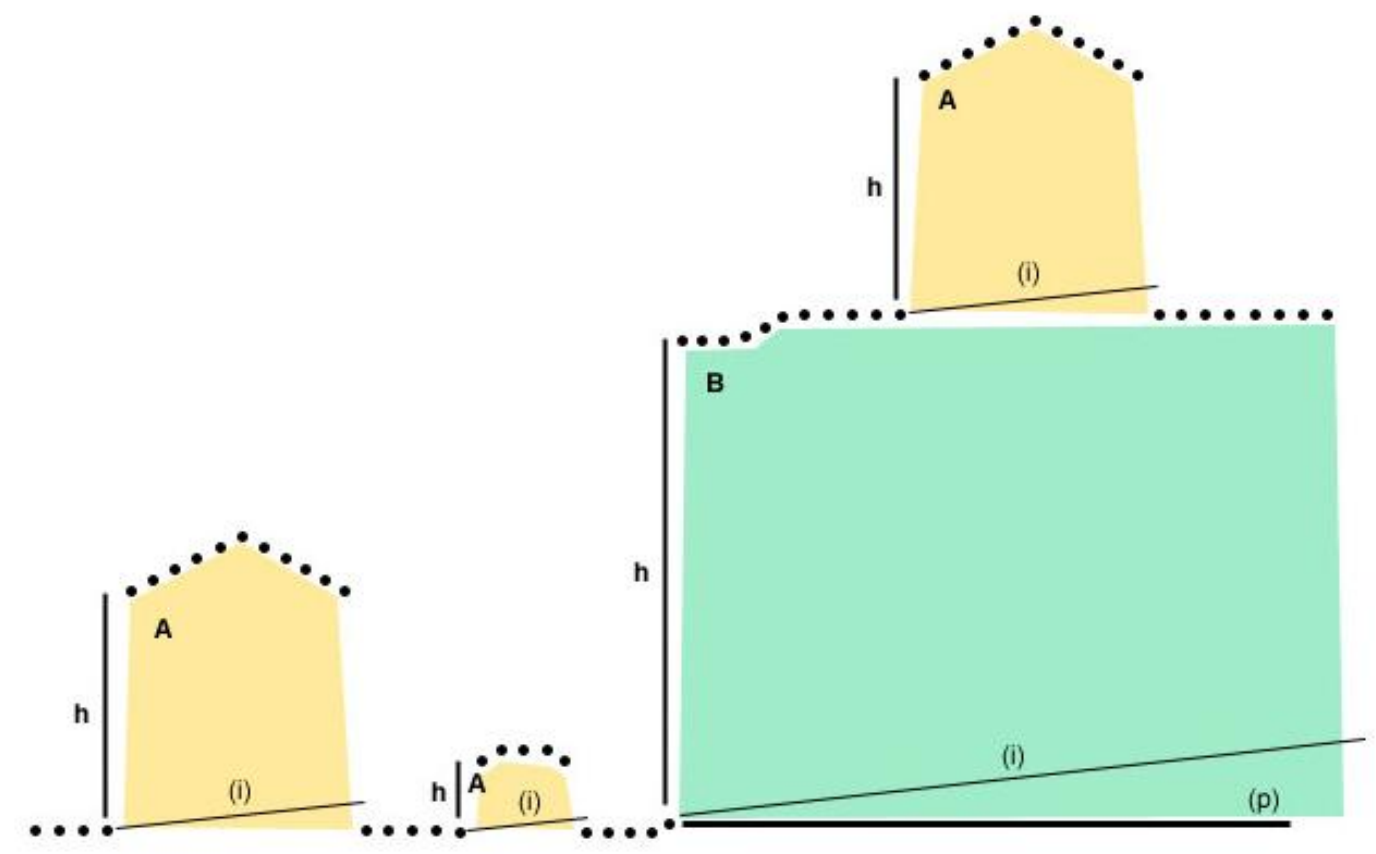

Figura 4. Exemplo de uma área de filtragem; (i) linha de inclinação provável do terreno, com base em um ponto de terreno; (p) linha do limite de propagação da regra da inclinação, partindo do início da linha de inclinação; (A) objetos sobre o terreno, como casas, carros, etc.; (B) áreas de relevo natural, como vales e montanhas, por exemplo; (h) diferença de altura entre dois pontos.

A primeira etapa do algoritmo é detectar os primeiros pontos não pertencentes ao terreno. É necessário o conhecimento da região de interesse dado os pontos de origem. Essa região é definida como a região de adjacência do ponto, e esta é usada para determinar se este ponto é pertencente ou não ao terreno.

Os dois procedimentos que decidem se um determinado ponto pertence ou não ao terreno é apresentado em Algoritmo 1 e Algoritmo 2. O Algoritmo 1 recebe como 
parâmetro um conjunto $A$ de pontos adjacentes, um ponto $p$ de origem, e um valor de altura máxima definido por hmax. No algoritmo é verificado se existe algum ponto dentre os elementos do conjunto de adjacentes que tenha uma diferença de altura maior que hmax. A proposta do Algoritmo 2 é inversa a ideia apresentada no Algoritmo 1.

Algoritmo 1. Detecção dos pontos que não pertencem ao conjunto

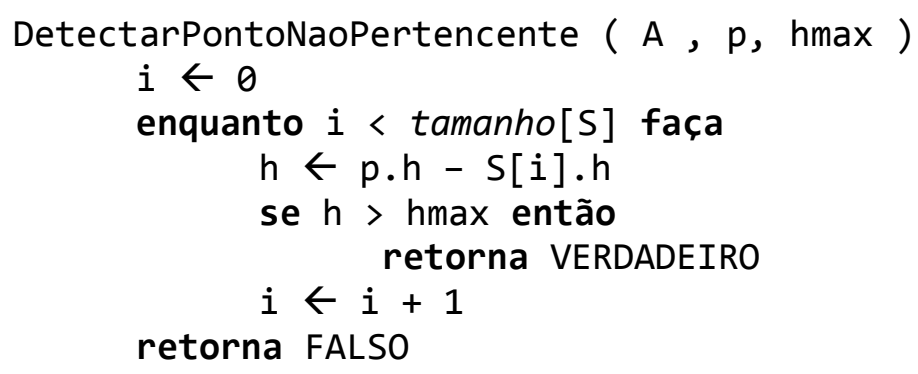

Algoritmo 2. Detecção dos pontos que pertencem ao conjunto

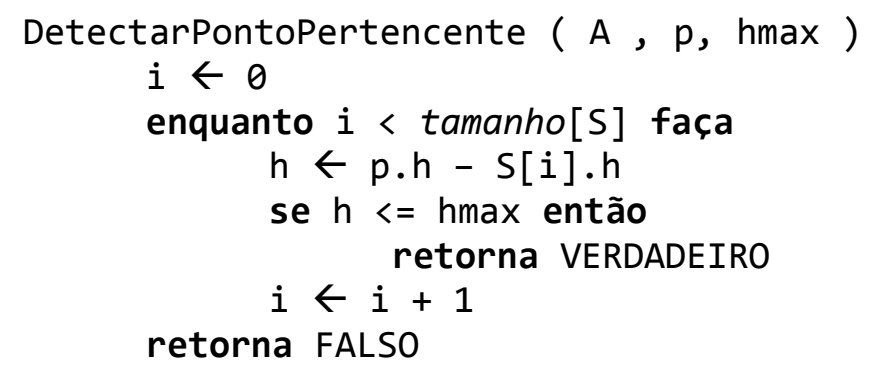

Se a declividade da região de análise for insignificante, é possível que o ponto de continuidade, encontrado pelo Algoritmo 2, nunca seja encontrado, ou venha a ser encontrado a uma distância muito grande, o que poderia gerar erros após a filtragem. Por conta disto, é estabelecido um limite de propagação, para que, caso não seja encontrado o ponto de continuidade do terreno a uma distância conhecida, nenhum ponto seja eliminado nessa região, a fim de evitar erros.

Assim, caso a propagação ultrapasse o valor de propagação pré-definido, o processo de busca, definido no Algoritmo 1, é continuado do estado seguinte. No Algoritmo 3 é apresentado um pseudocódigo do procedimento de filtragem na direção da altura da grade $\mathrm{G}$. O algoritmo que leve em consideração a direção de largura é semelhante ao Algoritmo 3. Após o processo 
de filtragem, os pontos que são considerados

não pertencentes ao terreno são eliminados.

Nas Figuras 4 e 5 é ilustrado o método de filtragem, em que nas áreas $A$, referente aos objetos, todos os pontos foram eliminados, e na área $B$, referente a um relevo natural do terreno, os pontos foram mantidos.

Algoritmo 3. Filtragem dos pontos não pertencentes ao terreno

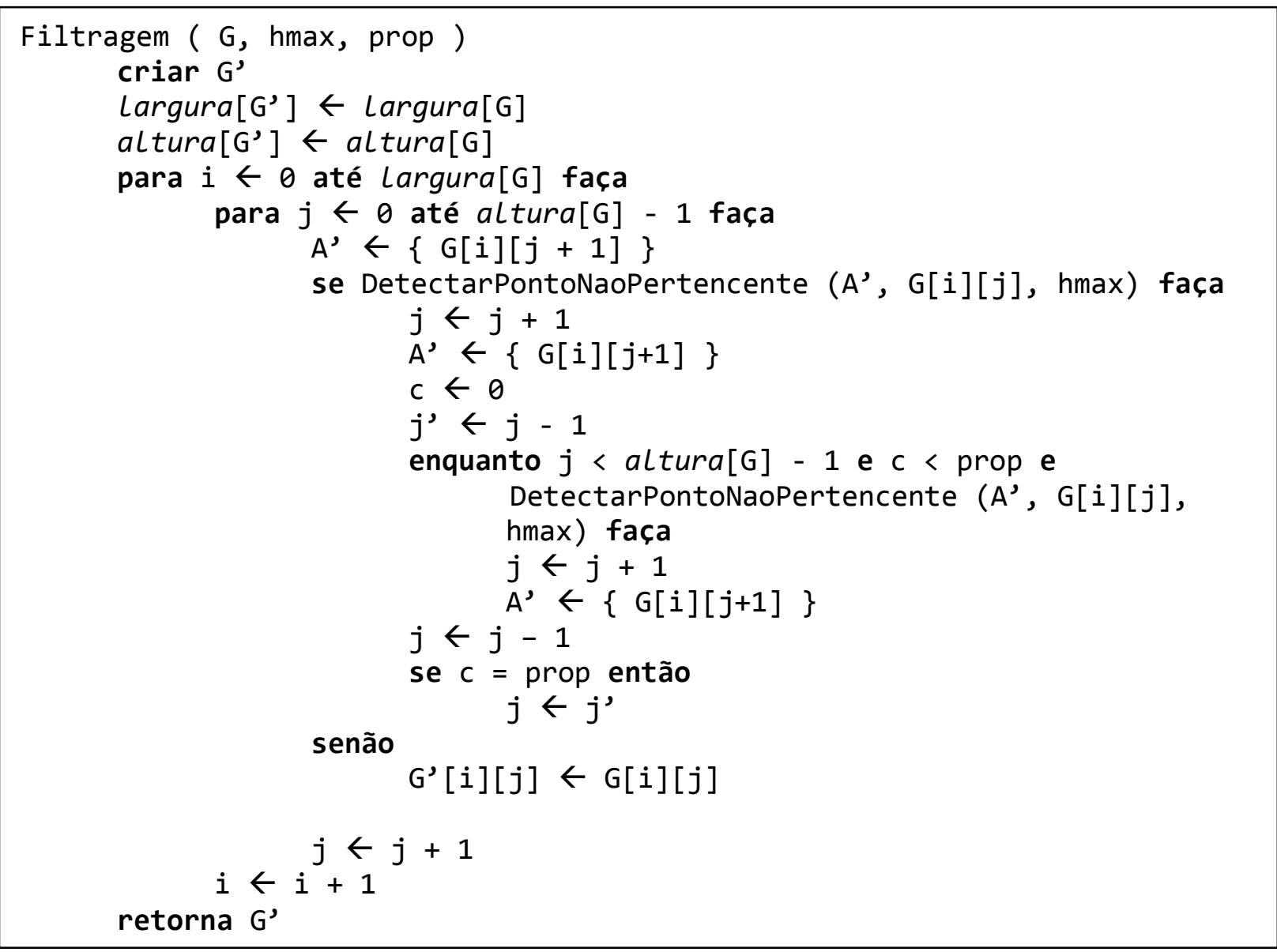




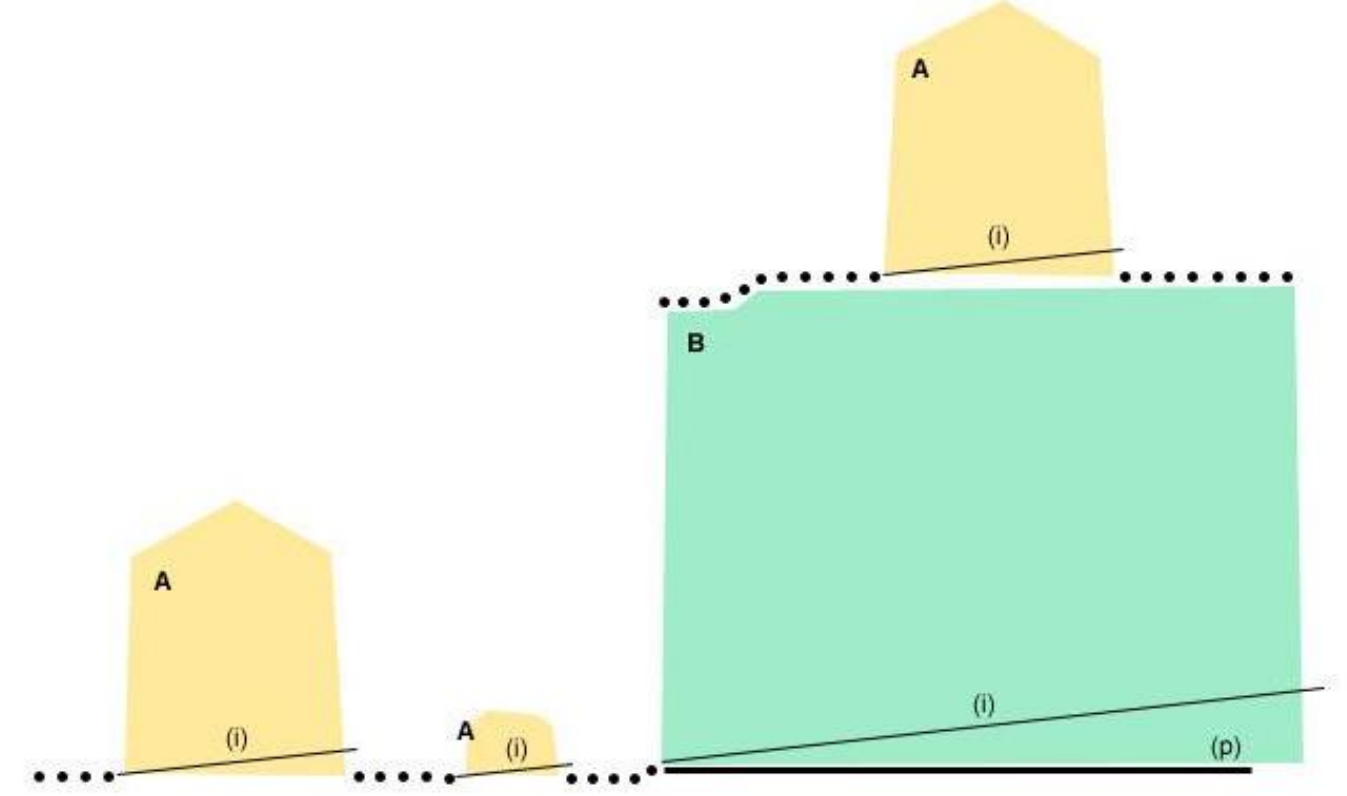

Figura 5. Exemplo de uma área de filtragem, com os pontos de região $A$ eliminados após a filtragem, seguindo a inclinação; (i) linha de inclinação provável do terreno, com base em um ponto de terreno; $(p)$ linha do limite de propagação da regra da inclinação, partindo do início da linha de inclinação; (A) objetos sobre o terreno, como casas, carros, etc.; (B) áreas de relevo natural, como vales e montanhas, por exemplo.

\section{TRABALHOS RELACIONADOS}

Sithole (2001) propôs um filtro que parte da superação dos filtros baseados na declividade utilizando $o$ gradiente de declinação como parâmetro para separar os pontos de terreno dos pontos não pertencentes ao terreno. O filtro proposto utiliza uma abordagem que lida com um plano de corte e um gerador de cones. O gerador de cones gera um cone tridimensional para um determinado ponto (as declividades do ponto em relação aos demais). Em seguida é estabelecido um valor $m$ que corresponde à altura do plano de corte. Se o plano de corte tocar o cone, o ponto é descartado. Nos primeiros experimentos a altura do plano de corte foi fixada. A partir dos primeiros experimentos, observou-se que os resultados eram melhores utilizando-se vários planos de corte específicos para cada ponto, por uma estimativa de valor de declividade mínimo (por hipótese, foi suposto que a menor altura numa certa região pertence ao terreno). Sithole (2001) conclui que o método não resolve o problema de filtragem em todos os casos (áreas urbanas e de vegetação), e que os resultados não foram tão bons quanto se esperava. 
Sithole e Vosselman (2005) propuseram um processo de filtragem com uso de segmentação da nuvem de pontos. Cada segmento é uma região em que os caminhos entre os pontos estão suavemente interligados. Por meio disso, criam-se vários contextos e informações (para cada segmento), e caminhos entre os segmentos, que são utilizadas para decidir se os pontos de determinado segmento são pertencentes ou não ao terreno. Os resultados para avaliação nesse trabalho foram realizados como estudo experimental. Sithole e Vosselman (2005) deixaram em aberto um velho problema em filtragem de MDE: como realizar a filtragem, com taxa de erro baixa, áreas de vegetação predominante, com altas taxas de irregularidade.

Alguns métodos de interpolação que retornam resultados satisfatórios para uma área específica pode não trazer bons resultados para outra área. Por exemplo, em áreas com mudanças suaves no nível de elevação da superfície, métodos baseados em altura máxima podem levar a resultados grosseiros, enquanto métodos baseados na média das alturas podem trazer um resultado melhor. O problema consiste em determinar o método apropriado para uma região de acordo com algumas regras (HOLLAUS et al., 2010).
Hollaus et al. (2010) propuseram um processo para realizar uma análise prévia, dos dados de entrada (nuvem de pontos), criar camadas de análise, selecionar a partir da análise alguns métodos de interpolação baseado em regras definidas por especialista. Com base nas informações de análise e dos métodos levantados, cria-se o modelo digital de terreno.

\section{METODOLOGIA}

Para a realização deste trabalho foram realizados experimentos com uso de um único arquivo de dados laser, representando uma região real da cidade de Curitiba, do estado do Paraná. Os resultados desse trabalho foram considerados como parte de um estudo experimental, cujo objetivo foi elaborar um método de filtragem eficiente. Para isso foram estudados diferentes métodos e técnicas de filtragem de dados, sendo que toda a análise foi feita de forma analítica.

\section{EXPERIMENTOS}

Foram realizados alguns experimentos em dados reais para avaliar o método de filtragem proposto. Todos os testes foram realizados sobre um conjunto de dados laser, armazenados em arquivo texto.

Na Tabela 1 é mostrado um exemplo da formatação do arquivo de dados laser, em 
que cada linha corresponde ao dado de um feixe de laser emitido, sendo a coordenada tridimensional, junto à intensidade da reflexão do material atingido pelo feixe, e caso exista, a informação de uma segunda reflexão.

Tabela 1. Exemplo da formatação do arquivo com dados laser.

\begin{tabular}{c|c|c|c|c|c|c|c}
\hline $\mathrm{x}$ & $\mathrm{y}$ & $\mathrm{z}$ & $\mathrm{i}$ & $\mathrm{x}$ & $\mathrm{y}$ & $\mathrm{z}$ & $\mathrm{i}$ \\
\hline 100.0 & 200.0 & 150.0 & 43 & & & & \\
\hline 101.1 & 210.2 & 151.0 & 57 & 101.1 & 211.3 & 141.6 & 8 \\
\hline 101.3 & 211.3 & 151.1 & 24 & & & & \\
\hline 101.5 & 210.9 & 150.9 & 28 & & & & \\
\hline 100.9 & 212.1 & 152.0 & 89 & 110.6 & 212.5 & 100.6 & 9 \\
\hline
\end{tabular}

O conjunto de pontos tridimensionais utilizado nos testes descritos nas seções seguintes é formado por todos os pontos de primeiro retorno dos pulsos de laser emitido à superfície. De cada retorno, apenas as coordenadas xyz foram utilizadas, descartando os valores de intensidade.

A Seção 5.1 apresenta como a grade regular foi gerada a partir do conjunto de pontos, a Seção 5.2 apresenta como foi realizada a suavização de ruídos na altimetria do conjunto de pontos. A Seção 5.3 apresenta como foi aplicado o algoritmo de filtragem.

\subsection{Geração da Grade}

O conjunto de pontos extraídos dos arquivos é denominado nuvem de pontos tridimensionais. Esse conjunto de pontos geralmente encontra-se irregularmente distribuído. É importante para o funcionamento do método que este conjunto esteja classificado (regular). Devido o uso de técnicas de processamento digital de imagens, tornou-se necessário a classificação da nuvem de pontos em uma grade regular. Inicialmente, para a geração da grade regular foi necessário definir uma dimensão de grade, sobre o conjunto de pontos. A dimensão definida é utilizada para definir os espaçamentos regulares nos eixos $x$ e $y$. Em seguida, todos os valores de espaçamento em $x$ e $y$ da grade tiveram seus valores de cota $z$ interpolados, seguindo a fórmula de interpolação por média simples apresentada na Seção 3, Equação 5.

Para definir a dimensão da grade, primeiramente foram obtidas as médias das diferenças entre as distâncias $x$ e $y$ de cada par de pontos adjacentes, sendo ambos os conjuntos ordenados e com valores distintos. 
As Equações 6 e 7 apresentam como as médias $(x, y)$ foram obtidas.

$$
\begin{aligned}
& u_{x}=\frac{\sum_{i=1}^{n}\left|x_{i}-x_{i+1}\right|}{n} \\
& u_{y}=\frac{\sum_{i=1}^{n}\left|y_{i}-y_{i+1}\right|}{n}
\end{aligned}
$$

Por fim, é definida a dimensão $(x, y)$, utilizando-se de das médias $u_{x}$ e $u_{y}$. As Equações 8 e 9 apresentam a formulação para definição das dimensões finais da grade.

$$
\begin{aligned}
& d_{x}=\frac{\left|x_{\max }-x_{\min }\right|}{u_{x}} \\
& d_{y}=\frac{\left|y_{\max }-y_{\min }\right|}{u_{y}}
\end{aligned}
$$

Os valores de cota $z_{x y}$ foram calculados utilizando-se do interpolador de média simples (Equação 3).

\subsection{Suavização dos Ruídos}

Após a interpolação dos valores de cota $z_{x y}$, há a possibilidade de que alguns pontos da grade estejam com seus valores significativamente altos em relação aos valores de sua vizinhança. Com a disposição do conjunto de pontos em uma grade regular foi possível a aplicação de técnicas de suavização de ruídos, com base nos conceitos de processamento digital de imagens.

O filtro utilizado foi o da mediana. Ao aplicar-se o filtro da mediana na grade regular, houve a suavização de algumas imperfeições do modelo. Em todos os testes, utilizou-se o filtro da mediana para a suavização dos valores de cota $z$ da grade com vizinhança $5 \times 5$.

\subsection{Aplicação do Método Proposto}

O algoritmo apresentado na Seção 2.4 necessitou de alguns parâmetros para a realização da filtragem. São eles: altura máxima (hmax), prop (propagação) e G (grade regular). Para fins de simplificação, os valores de cota $z$ da grade foram normalizados, para todos os testes, com base na Equação 10.

$$
z_{i}^{\prime}=\frac{z_{i}}{\left|z_{\max }-z_{\min }\right|}
$$

Foi considerada que a altura máxima da área real é igual a 110 metros, definida empiricamente, tomando como base os dados provenientes da grade regular. Dado que os valores de $\operatorname{cota} z$ da grade estão normalizados no intervalo de 0 a 1 , cada parâmetro foi calculado com base na estimativa da altura máxima da área real.

Para uma altura máxima de 1 metro entre dois pontos na vizinhança, com distância de 1 metro em si, obtêm-se hmax por meio da Equação 11. 


$$
\Delta h_{\max }(1 m)=\frac{1}{110} \cong 0.009090
$$

Definiu-se que um objeto não pertencente ao terreno deveria ter sua descontinuidade inferior a 300 metros. Assim, o valor de propagação (prop) foi definido como de 300 metros.

Os valores dos parâmetros apresentados nesta seção foram os valores utilizados no experimento.

\section{ANÁLISE E RESULTADOS}

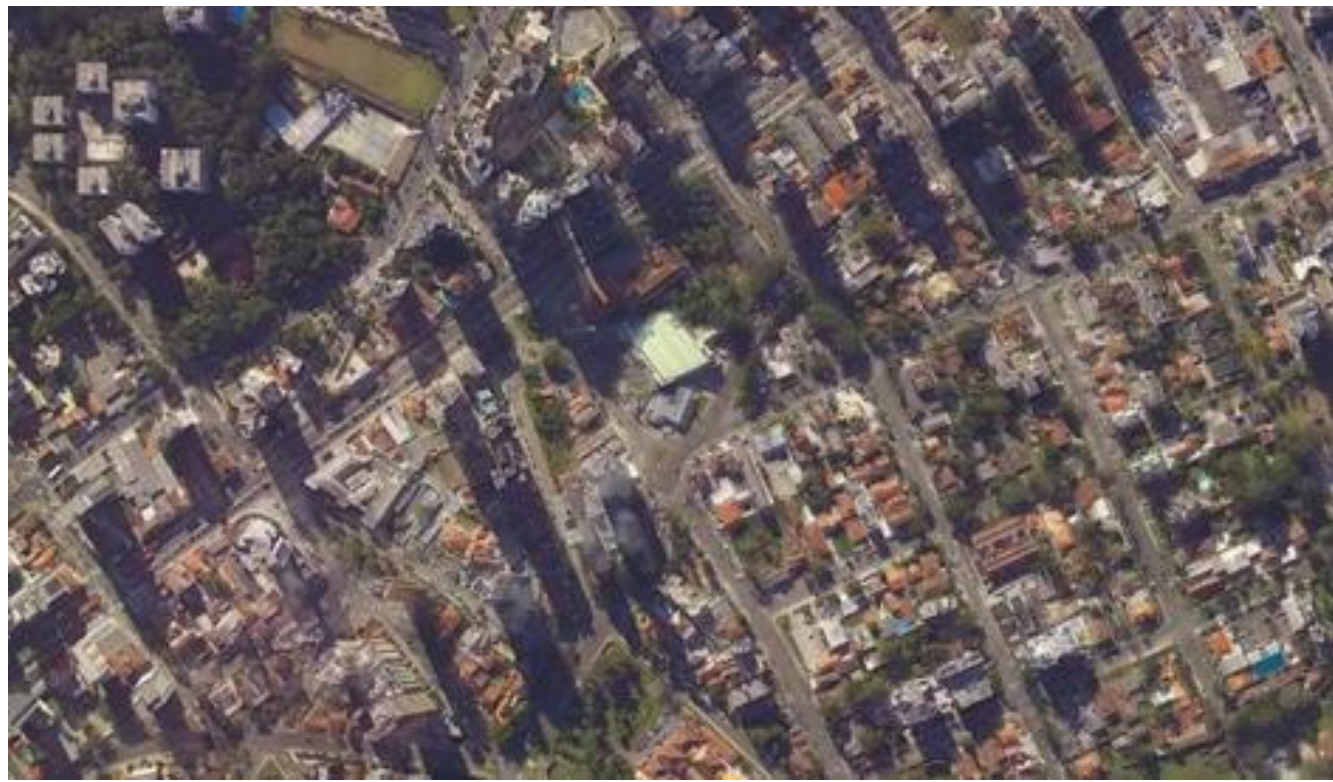

Figura 6. Imagem aérea de parte da região de área varrida pelo sistema de varredura a laser aerotransportado.

A definição da altura máxima da área total é derivada por suposição, com base em um valor estimado e aproximado da altura de um prédio de 30 a 40 andares. Na Figura 8 é
Com base na Figura 6 é possível verificar a presença de muitas árvores, prédios, e um grande número de casas na região da imagem. A Figura 6 é uma foto da região real, de onde o sistema de varredura a laser aerotransportado coletou os dados de altimetria utilizados para a análise. As Figuras 6 e 7 estão aproximadamente alinhadas na mesma região de interesse. Na Figura 7 é mostrado um modelo tridimensional projetado a partir da grade regular de pontos. 
proposto foi aplicado em quatro sentidos na grade: da direita para a esquerda, da esquerda para a direita, de cima para baixo e de baixo para cima. Assim, o Algoritmo 3, apresentado na Seção 2.4 foi levemente modificado para atender ao experimento. $\mathrm{Na}$ Figura 9 é mostrada uma imagem binária após a filtragem, na qual é possível observar todos os pontos considerados não pertencentes ao terreno.
Após a identificação dos pontos a serem eliminados do modelo, como ilustrado na Figura 9, foi feita uma recomposição da forma do terreno. Na Figura 10 é mostrada uma imagem em perspectiva, da mesma região apresentada na Figura 8, que contém os pontos considerados não pertencentes ao terreno. Nesta figura é possível observar a presença de muitas edificações, como prédios e casas.

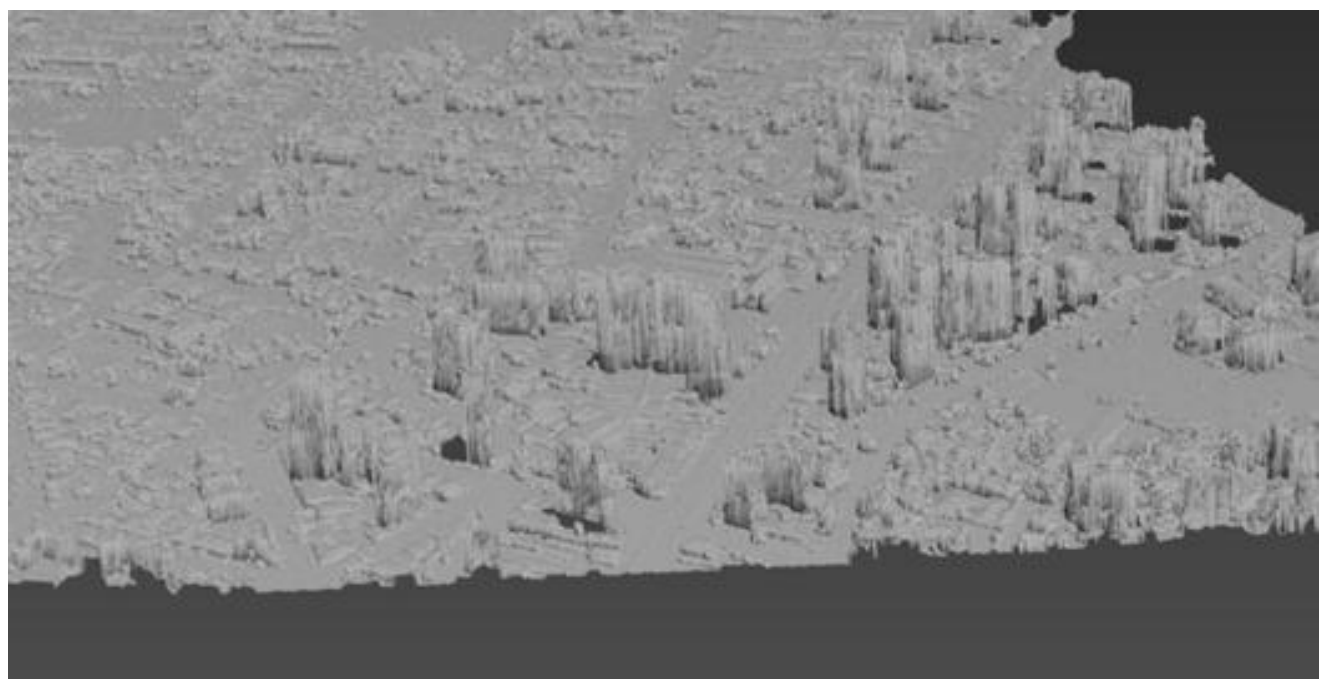

Figura 7. Imagem em perspectiva do modelo tridimensional da grade regular, aproximadamente alinhado com a imagem apresentada na Figura 6.

A reconstrução do modelo foi feita utilizando novamente o filtro da mediana, utilizado inicialmente para suavização da altimetria da grade. Entretanto, a vizinhança utilizada precisou ser ampliada, devido ao tamanho dos buracos produzidos pela filtragem. Foi definida uma vizinhança de $25 \times 25$ para o filtro da mediana, aplicado somente nas áreas em que houve pontos ausentes. Na Figura 11 é apresentado o resultado após a reconstrução com o filtro mediana de vizinhança $25 \times 25$.

Com isso, obteve-se um MDT gerado a partir de um MDS, estruturado em uma grade regular. Não é garantido que o método desenvolvido gere um modelo isento de erros e também não houve como analisar com precisão a quantidade de erros gerados. 
Analisando a Figura 11, analiticamente, é possível perceber algumas imperfeições no modelo, porém, os resultados obtidos são considerados satisfatórios para nossa pesquisa. Na Figura 12 são apresentados os estados de uma mesma região durante o processo de filtragem, iniciando em (a) com o modelo bruto e finalizando em (c) com o MDT gerado.

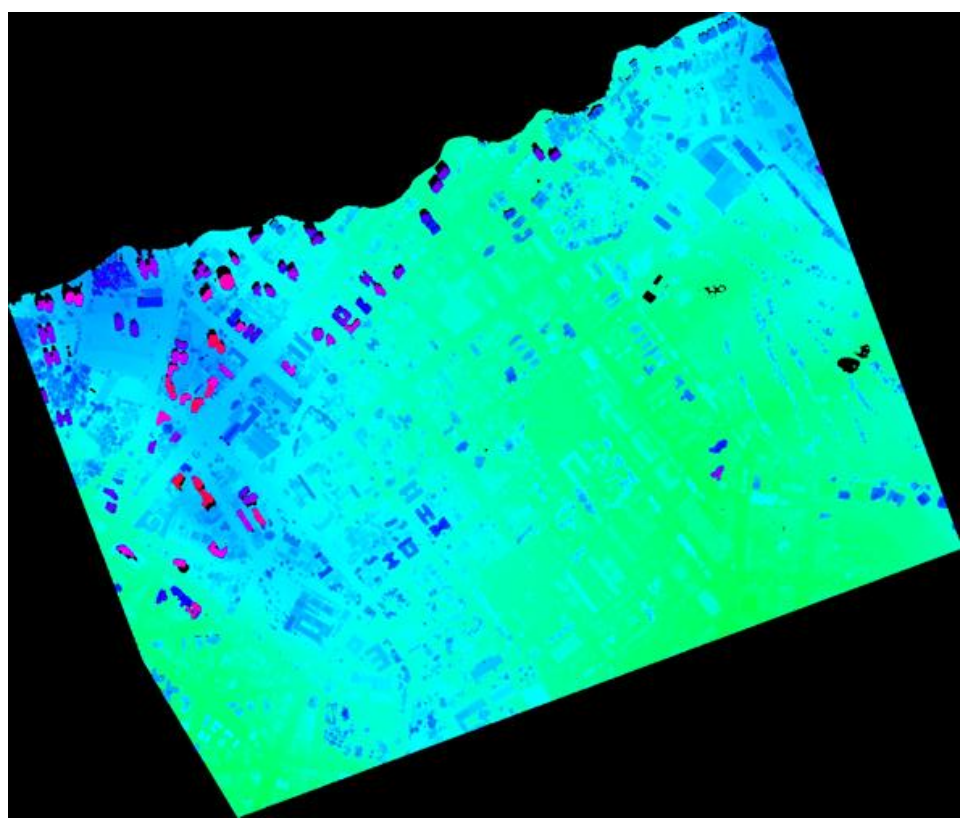

Figura 8. Imagem topográfica da grade regular, após aplicação do filtro da mediana, com vizinhança $5 \times 5$.

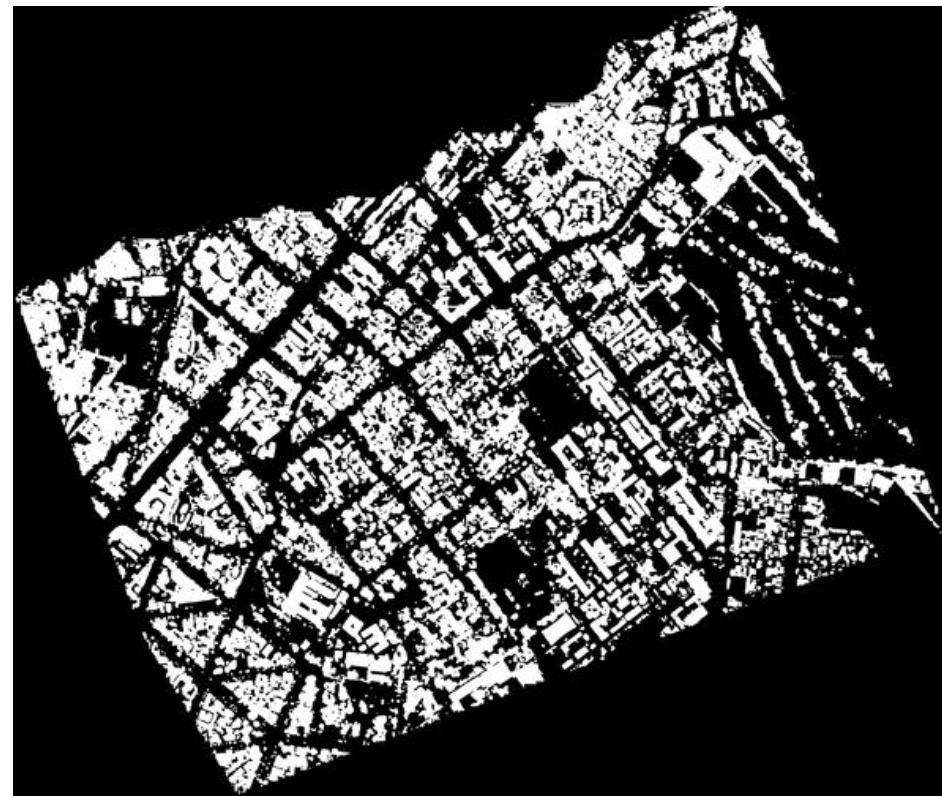

Figura 9. Imagem binária da grade, dos pontos considerados não pertencentes ao terreno, após a aplicação do método de filtragem (Algoritmo 3). 


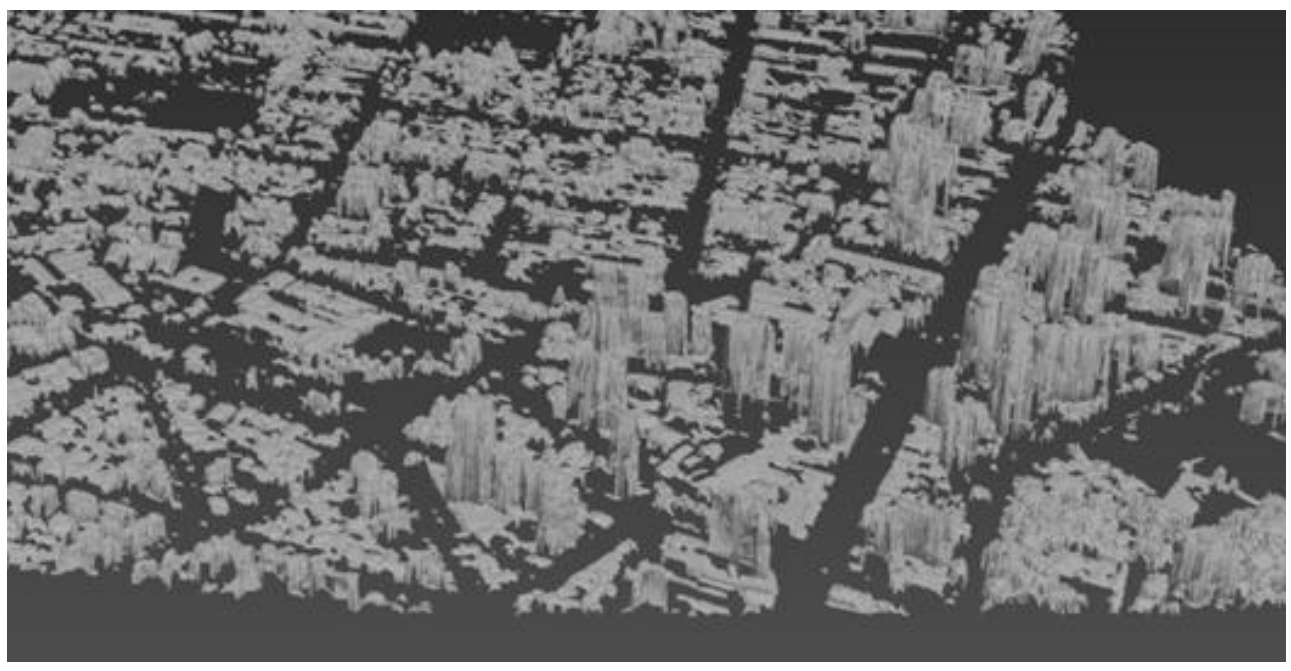

Figura 10. Imagem em perspectiva de parte da região de área total, apenas com os pontos eliminados pela filtragem, da região apresentada na Figura 7.

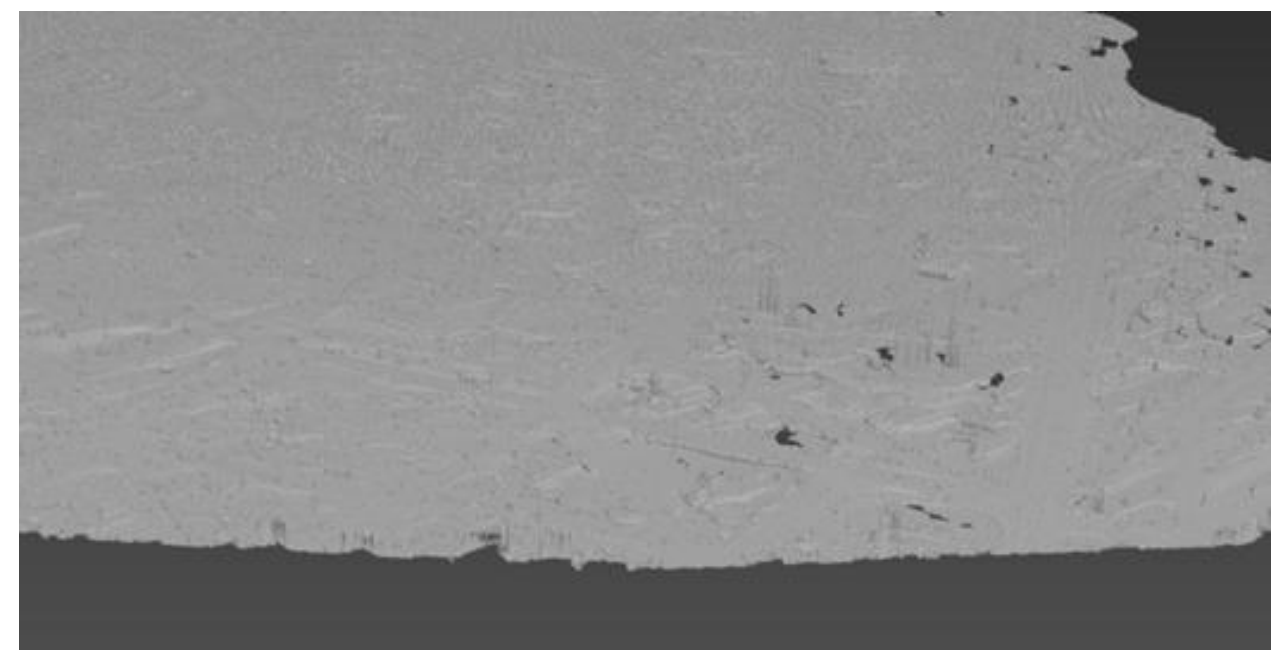

Figura 11. Imagem em perspectiva de parte da região de área total, do modelo digital de terreno, após a aplicação do filtro da mediana, da região apresentada na Figura 7.

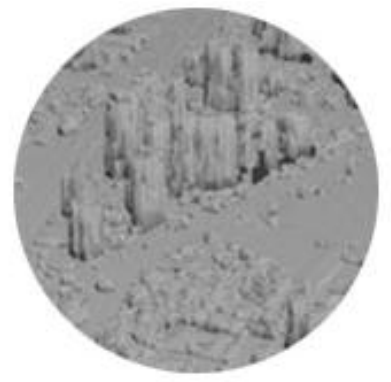

(a)

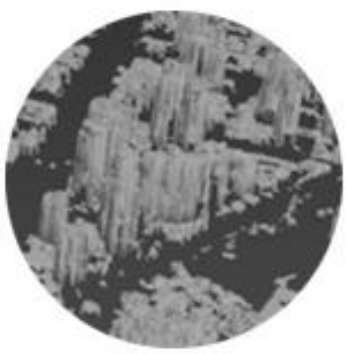

(b)

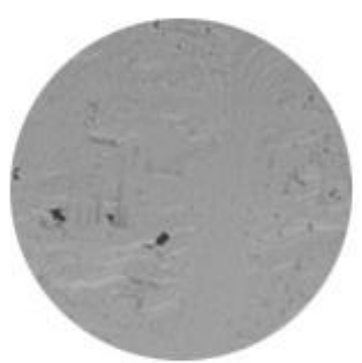

(c)

Figura 12. Demonstração das etapas de filtragem no modelo. (a) é a região em grade regular, antes de processo de filtragem. (b) é a região dos pontos eliminados. (c) é a região sem os pontos não pertencentes ao terreno, formando o MDT. 
Como não foi feito nenhum tipo de comparação com outros métodos propostos por outros autores da literatura relacionada, houve uma dificuldade para validação dos resultados. Espera-se que as pesquisas a serem desenvolvidas a partir desta venham estabelecer um critério de mensuração, para comparar com os resultados apresentados por outros autores. Os resultados e análise desta pesquisa tratam de um estudo experimental apenas.

\section{CONCLUSÕES}

Os sistemas de varredura a laser são muito úteis para obtenção de dados tridimensionais da superfície terrestre, devido a sua alta capacidade de aquisição rápida e precisa do conjunto de pontos de elevação. Dentre os tipos de classificação do conjunto de pontos gerado pela varredura a laser, a classificação em grade possibilita o aproveitamento de algumas técnicas consolidadas em processamento digital de imagens.

O método desenvolvido apresentou resultados analiticamente satisfatórios quanto aos princípios básicos de filtragem. É importante ressaltar que a definição correta dos parâmetros, com base no conhecimento prévio das características do terreno, é um diferencial para a geração de um MDT com baixa quantidade de erros.
Todo o experimento e análise foram feitos empiricamente, dificultando uma mensuração precisa dos erros gerados. O método proposto foi capaz de, a partir de um conjunto de dados com pontos de coordenadas tridimensionais, filtrar e gerar um MDT, observado analiticamente.

As pesquisas realizadas nessa área de conhecimento evoluem juntamente aos avanços tecnológicos, assim, os métodos vêm sendo gradativamente aprimorados, com o objetivo de se obterem bons resultados, com precisão e com menor quantidade de erros.

Pesquisas futuras poderão ser realizadas no sentido de aperfeiçoar o método proposto, possibilitando sua aplicação em outras fontes de dados, com objetivo de diminuir a quantidade de erros, gerando modelos digitais de terreno próximos aos reais.

\section{REFERÊNCIAS}

BARBOSA, R.L.; SILVA, J.F.C. Geração de Modelo Digital do Terreno por aproximações sucessivas utilizando câmaras digitais de pequeno formato. 1999. 97f. Dissertação (Mestrado) - Universidade Estadual Paulista, São Paulo.

CENTENO, J.A.S.; MITISHITA, E.A. Laser scanner aerotransportado no estudo de áreas urbanas: a experiência da UFPR. In: SIMPÓSIO BRASILEIRO DE SENSORIAMENTO REMOTO, 13., 2007. Anais... Florianópolis, Brasil, 21-26 abril 2007, INPE, p. 3645-3652. 
ELMIRO, M.A.T. et al. Avaliação de Modelos Digitais de Terrenos e Modelos Digitais de Superfície utilizando metodologia InSAR de duas frequências. 2003. Disponível em: http://www.cartografia.org.br/xxi_cbc/008SR02.pdf. Acesso em: 12 dez. 2012.

FAZAN, A.J.; DAL POZ, A.P. Extração de contornos de telhado de edifícios no espaçoobjeto baseada em snakes e programação dinâmica a partir de TIN/LASER e imagens aéreas de alta resolução. 2011. 120f. Tese (Doutorado) - Departamento de Cartografia Faculdade de Ciências e Tecnologia, Universidade Estadual Paulista "Júlio de Mesquita Filho", Presidente Prudente.

\section{FELGUEIRAS, C.A. Modelagem numérica de} terreno. 2012. Disponível em: http://www.dpi.inpe.br/gilberto/tutoriais/gis _ambiente/4mnt.pdf. Acesso em: 25 set. 2012.

GDAL: GDAL GRID Tutorial. 2012. Disponível em: http://www.gdal.org/grid_tutorial.html. Acesso em: 10 nov. 2012.

GONZALEZ, R.C.; WOODS, R.E. Digital image processing. Reading, Massachusetts: Addison-Wesley, 1993.

HOLLAUS, M. et al. Land cover dependent derivation of digital surface models from airbone laser scanning data. International Archives of Photogrammetry and Remote Sensing, Saint-Mandé France, v.XXXVIII, n.3A, p.1-3, 2010.

SITHOLE, G. Filtering of laser altimetry data using slope adaptive filter. International Archives of Photogrammetry and Remote Sensing, Annapolis, v.XXXIV-3/W4, p.22-24, 2001.

SITHOLE, G.; VOSSELMAN, G. Filtering of airborne laser scanner data based on segmented point clouds. International Archives of Photogrammetry and Remote
Sensing, Enschede, Holanda, n.3, p.12-14, 2005.

TERRA IMAGING. Laser scanning. 2013. Disponível em: http://www.terraimaging.de/index.php/en/t echnologie/laserscanning. Acesso em: 05 nov. 2013.

VOSSELMAN, G. Slope based filtering of laser altimetry data. International Archives of Photogrammetry, Remote Sensing and Spatial Information Sciences, Amsterdam, Netherlands, v.33, n.B3/2, p.935- 942, 2000. 